\title{
Mutation of EGFR in Non-small Cell Lung Cancer, a Regional Study in Upper Egypt
}

\author{
Samir Shehata Mohammed Eid ${ }^{1}$, Ahmed Roshdi Hamed Ahmed ${ }^{2, *}$, \\ Marwa Ismail Khalaf Abdelgawad ${ }^{1}$, Ahmed El-Sayed Mohamed ${ }^{3}$, Amany Osama Mohamed $^{4}$, \\ Dalia Osama Mohamed ${ }^{5}$, Mona Mahmoud Sayed ${ }^{5}$, Ebtesam Mahmoud Aly Elgezawy ${ }^{6}$, \\ Heba Mohammed Saad-EIdeen ${ }^{7}$
}

${ }^{1}$ Department of Clinical Oncology, Assiut University, Assiut, Egypt

${ }^{2}$ Department of Pathology, Sohag University, Sohag, Egypt

${ }^{3}$ Department of Clinical and Radiation Oncology, Sohag University, Sohag, Egypt

${ }^{4}$ Department of Medical Biochemistry, Assiut University, Assiut, Egypt

${ }^{5}$ Department of Radiation Oncology, South Egypt Cancer Institute, Assiut, Egypt

${ }^{6}$ Department of Immune-Hematology, Regional Laboratory and Central Blood Bank, Aseer Region, KSA

${ }^{7}$ Department of Anatomy, College of Medicine, Jouf University, Jouf, the Kingdom of Saudi Arabia

\section{Email address:}

samir_eid@hotmail.com(S. S. M. Eid), ahmed_ahmed4@med.sohag.edu.eg (A. R. H. Ahmed), esmailmarwa@yahoo.com (M. I. K. Abdelgawad),dr_ahmed_sayed76@yahoo.com (A. El-Sayed M.), amanyosama@yahoo.com (A. O. Mohamed),daliao.mohamed2018@gmail.com(D. O. Mohamed),mmsaamz@yahoo.com (M. M. Sayed), eelgezawy1@yahoo.com (E. M. A. Elgezawy), hebasaadeldien2015@yahoo.com (H. M. Saad-Eldeen)

${ }^{*}$ Corresponding author

\section{To cite this article:}

Samir Shehata Mohammed Eid, Ahmed Roshdi Hamed Ahmed, Marwa Ismail Khalaf Abdelgawad, Ahmed El-Sayed Mohamed, Amany Osama Mohamed, Dalia Osama Mohamed, Mona Mahmoud Sayed, Ebtesam Mahmoud Aly Elgezawy, Heba Mohammed Saad-Eldeen. Mutation of EGFR in Non-small Cell Lung Cancer, a Regional Study in Upper Egypt. Cancer Research Journal.

Vol. 8, No. 1, 2020, pp. 1-7. doi: 10.11648/j.crj.20200801.11

Received: December 2, 2019; Accepted: December 26, 2019; Published: January 7, 2020

\begin{abstract}
Management of non-small cell lung cancer has been changed dramatically since detection of EGFR mutation. The rate of EGFR mutation in Upper Egypt is unknown. In this study; EGFR mutation in non-small cell lung cancer tissue was evaluated and correlated with clinicopathological features of the tumors. Tissue samples of non-small cell lung cancer that had been confirmed by histopathological and immunohistochemical examinations were evaluated for mutation of EGFR by StripAssay. Thirty four patients with non-small cell lung cancer; 21 males and 13 females with a mean age of 55.94 years were included in this study. The median tumor size was $6.0 \mathrm{~cm}$ and more than half of the tumors were classified as T3. Fourteen patients had N2 nodal stage and 19 patients had either malignant pleural effusion or distant metastasis. The majority of the tumors were adenocarcinomas and half of them were grade II. The two year survival rate of the patients was $70.6 \%$ and the overall survival was $58.8 \%$. EGFR mutation was recorded in $44.1 \%$ of the tumors; all of which were adenocarcinoma variant. The mutation was significantly more frequent in large sized tumors and it was relatively associated with poor survival. Mutation of EGFR was not associated with patients` age, sex, tumor grade, T stage, N stage or status of distant metastasis. The rate of EGFR gene mutation in non-small cell lung cancer in Upper Egypt is relatively high and target therapy based on these mutations could improve patients`outcome.
\end{abstract}

Keywords: NSCLC, EGFR Mutation, Adenocarcinoma, Tumor Size 


\section{Introduction}

Lung cancer is the third leading cause of cancer death worldwide. For a long time, lung cancer was classified as an incurable disease until innovation of new diagnostic and therapeutic tools. Incorporation of diagnostic molecular tools paved a great progress in cancer management particularly in non-small cell cancer; NSCLC [1]. According to data of the Egyptian National Cancer Registry Program, lung cancer is ranked as the fourth most frequent malignant neoplasm among men representing $5-7 \%$ of malignant tumors recorded between 2008 and 2011. The frequency of lung cancer has a geographical variation among Egyptian males being higher in Upper Egypt representing 7.63\% of cancers diagnosed in the same period versus $5.47 \%$ in Lower Egypt [2].

NSCLC displays several genetic abnormalities including epidermal growth factor receptor (EGFR), v-Ki-ras2 Kirsten rat sarcoma viral oncogene (KRAS) and anaplastic lymphoma kinase (ALK) mutations. Analysis of genes harboring concurrent mutations allowed better understanding of pathogenesis of this disease and hence identifying novel therapeutic targets that improve outcome of lung cancer [3]. The family of EGFR includes four tyrosine kinase receptors: HER-1 (EGFR), HER-2/neu (ERBB2), HER-3 (ERBB3), and HER-4 (ERBB4). EGFR is a trans-membrane receptor; activation of which initiates intracellular signaling cascade with subsequent expression of genes responsible for cell growth, survival, proliferation, differentiation and angiogenesis. It has been reported that deregulation of EGFR signaling pathway is involved in progression of lung cancer [4].

Discovery of EGFR gene has explained why some patients have an excellent response to oral tyrosine kinase inhibitor (TKI) of this receptor [5, 6]. NSCLC patients with Asian ethnicity, females, none smokers, and adenocarcinoma histology showed the highest clinical benefit when treated with EGFR-TKIs; namely gefitinib and erlotinib [6, 7]. The most frequent mutations of EGFR gene involve exon 18, 19, and 21. They are either point mutations (in exon 18 and 21) or deletions in (exon 19) clustered around intracellular tyrosine kinase ATP- domain. Analysis of domains of the mutant EGFR revealed reduced affinity for ATP in EGFR-TKI existence compared to wild type receptor. This existence correlates with efficiency of gefitinib and erlotinib tyrosine kinase inhibitors [5, 8].

Current guidelines consider EGFR-TKIs as a first-line treatment for patients with advanced NSCLC who harbor EGFR mutation, replacing the conventional cytotoxic chemotherapy $[7,9,10]$. Therefore, evaluation of EGFR mutation is necessary in clinical practice [11]. Data concerning EGFR mutations among NSCLC patients in Egypt is generally lacking with no available records about status of these mutations in Upper Egypt. This study was conducted to explore EGFR mutation in NSCLC and its relationship with clinicopathological features of this malignant neoplasm in Upper Egypt.

\section{Materials and Methods}

\subsection{Tissue Samples}

Thirty-four tissue samples of NSCLC patients admitted at Assiut University Hospitals, Sohag University Hospitals and South Egypt Cancer Institute between March 2016 and December 2017 were recruited for testing of EGFR mutations. Ethical approval to perform this work was obtained from Research Ethical Committees of the participating institutes. All tissue samples were obtained for diagnostic purposes by image-guided tru-cut needle biopsies. Different clinical and imaging parameters were obtained from patients' medical records and all tissue samples were reviewed and verified by a single pathologist. DNA extraction and PCR techniques were conducted at Tissue Culture and Molecular Biology Centre, Assiut University.

\subsection{EGFR StripAssay}

Mutations of exons number 18, 19, 20 and 21 of EGFR gene were detected by PCR and reverse hybridization technique using EGFR XL StripAssay kit (Vienna Lab Diagnostics, Austria). The oligonucleotides probes of EGFR StripAssay target three mutations in exon 18, 24 mutations in exon 19, one mutation in exon 20 and two mutations in exon 21 of EGFR gene (Table 1). DNA was extracted from formalin-fixed paraffin-embedded (FFPE) tissue sections using DNA FFPE tissue kit (Qiagen) according to manufacturer protocol. The concentration of the extracted DNA was measured by a spectrophotometer before storage at $-20^{\circ} \mathrm{C}$. Specifically hybridized biotinylated oligonucleotides are visualized using streptavidin-alkaline phosphatase and colored substrates.

\subsection{Immunohistochemical Subtyping of the Investigated Tumors}

Histopathological subtype of the investigated tumors was confirmed by evaluation of TTF1 expression, a tissue marker of pulmonary adenocarcinoma and CK5/6 expression, a tissue marker of squamous cell carcinoma by immunohistochemistry. Four micrometer-thick sections were de-paraffinized in xylene and rehydrated before washing thoroughly in running water. The endogenous peroxidase activity was blocked by incubation of tissue section in dual endogenous enzyme blocking solution (Dako Code K4065, Aligent, USA) for $10 \mathrm{~min}$ followed by washing in running water. Antigens were retrieved by boiling tissue sections in citrate buffer (10mM, pH 6.0) using a microwave for $10 \mathrm{~min}$. The sections were washed in phosphate-buffered saline (PBS) pH 7.6 before incubation with either mouse monoclonal anti-human TTF1 antibody (Dako, clone 8G7G3/1, Aligent, USA) or mouse monoclonal anti-human CK5/6 antibody (Dako, clone D5/16 B4, Aligent, USA) for one hour at room temperature. After washing in PBS, the sections were incubated with Peroxidase labeled polymer conjugated to goat anti-mouse immunoglobulin (Dako, code K4065, Aligent, USA) for $30 \mathrm{~min}$ at room temperature. The sections 
were washed with PBS and exposed to a freshly prepared 3,30-diaminobenzidine tetrahydrochloride (DAB) solution for 5-10 minutes to visualize brown deposits. The sections were counterstained by hematoxylin, dehydrated in graded alcohol and mounted as usual. TTF1 was visualized as nuclear brown staining while CK5/6 was demonstrated as cytoplasmic brown staining.

\subsection{Statistical Analysis}

The data were analyzed by IBM-SPSS (V 22.0) for
Windows. Probabilities of $<0.05$ were considered significant. The frequency of a categorical observation was measured by Chi-Square test $(\chi 2)$. Spearman's rho test was used to evaluate association among study groups and Mann-Whitney $\mathrm{U}$ test was performed to measure differences between study groups. Survival tables were used to calculate median survival rate of the patients and Kaplan Meier survival analysis was performed to compare overall survival among different study groups.

Table 1. Mutations of EGFR genes targeted by StripAssay.

\begin{tabular}{llll}
\hline EGFR exon number & Mutations & & \\
\hline Exon 18 & G719A & G719C & G719S \\
& K745_E749del & E746_A750del & E746_A750delinsIP \\
& E746_A750del & E746_T751delinsIP & E746_T751del \\
& E746_T751delinsA & E746_T751delinsV & E746_T751delinsVA \\
Exon 19 & E746_S752delinsI & E746_S752delinsA & E746_S752delinsV \\
& E746_S752delinsD & E746_P753delinsVS & L747_E749del \\
& L747_A750delinsP & L747_A750delinsP & L747_T751delinsP \\
Exon 20 & L747_T751delinsS & L747_T751del & L747_S752del \\
Exon 21 & L747_S752delins & L747_P753delinsQ & L747_P753delinsS \\
\hline
\end{tabular}

\section{Results}

\subsection{Patients`Data}

Thirty-four patients with non-small lung carcinomas were retrieved for this study including 21 males and 13 females. The patients' age ranged between 30 to 77 years with a mean $( \pm \mathrm{SD})$ of $55.94( \pm 12.34)$ years and a median of 60.00 years. The majority of the patients $(n=30)$ presented with cough, dyspnea or combined cough and dyspnea while associated hemoptysis was reported in eight patients. Bone pain and disturbed level of consciousness were the leading symptoms in three and one patients, respectively. Right lung was involved in 16 cases while left lung was affected in the remaining cases. The tumor masses were classified radiologically as central or peripheral in 23 and 11 patients, respectively and tumor size ranged between 2.0 and $10.0 \mathrm{~cm}$ with a mean $( \pm \mathrm{SD})$ and median values of $5.87( \pm 2.26)$ and $6.0 \mathrm{~cm}$, respectively. More than half of the tumors $(n=20)$ were classified as T3 while T1 and T4 were stated in 5 and 9 cases, respectively. Hilar and mediastinal lymphadenopathy were absent radiologically $(\mathrm{N}=0)$ in 12 cases while $\mathrm{N} 1, \mathrm{~N} 2$ and N3 stages were recorded in 7,14 and 1 patients, respectively. Distant metastasis was absent in 15 patients while metastatic deposits and/or malignant pleural effusion of contralateral lung were recorded in 5 patients. Distant metastasis was demonstrated in 14 cases; involving liver, suprarenal glands, bone, brain and Virchow's lymph node in 3, 2, 2, 1 and 1 patients, respectively and involving multiple sites in 5 patients.

\subsection{Tumors`Characteristics and EGFR Mutations}

The tumors were classified as adenocarcinomas (Figure 1A) and undifferentiated carcinomas (Figure 1B) in 26 and 8 lesions; respectively. Adenocarcinoma phenotype was confirmed by TTF1 protein expression (Figure 1C). Based on immunohistochemical evaluations of TTF1 and CK5/6 molecules (Figure 1D), undifferentiated neoplasms were classified as pulmonary adenocarcinomas or squamous cell carcinomas in 5 and 3 tumors, respectively. The tumors were graded as I, II, III, and IV in 2, 16, 8 and 8 cases, respectively.

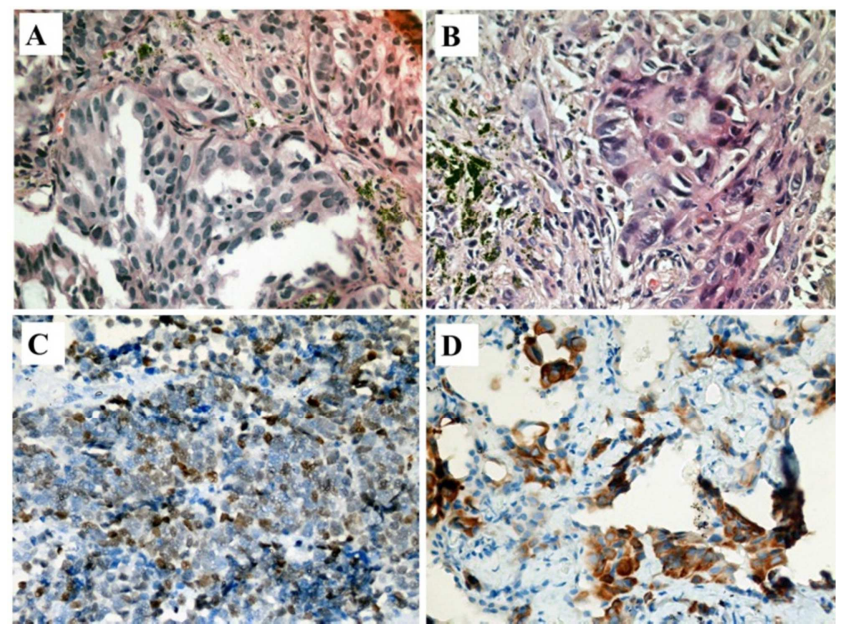

Figure 1. Histological sections of adenocarcinoma (A) and large cell carcinoma (B) of the lung and expression of TTF1 (C) and CK5/6 (D) molecules. $H \& E$ ( $A$ and $B$ ) and immunohistochemical $(C$ and $D)$ stained sections; original magnification is X400 for $A$ and $B$ and X200 for $C$ and D.

Mutation of EGFR (Figure 2) was demonstrated in 15 tumors representing a significant proportion of the investigated cases $(44.1 \%)$. EGFR mutations were located in chromosome 18, 19, 20 and 21 in 4,1, 1 and 3 tumors, respectively. Furthermore, four tumors harbored combined EGFR mutation in chromosomes 20 and 21 and two tumors 
harbored combined EGFR mutations in chromosomes 19 and 20. Statistically, EGFR mutation was not associated with patients' age or sex. There was no association of EGFR mutation with tumor site, histological type, grade or stage. Additionally, mutations of EGFR were not associated with nodal stage or status of distant metastasis (Table 2). On the other hand, EGFR mutation is significantly associated with tumor size (Spearman's rho $=-3.45, \mathrm{p}<0.05$ ). The larger the tumor size, the more frequent mutations of EGFR (Mann-Whitney, $\mathrm{p}=0.047$ ).

Table 2. Association of EGFR mutation with different clinic-radiological and pathological parameters.

\begin{tabular}{|c|c|c|c|c|}
\hline \multirow{2}{*}{ Parameter } & & \multicolumn{2}{|c|}{ EGFR status } & \multirow{2}{*}{ P value } \\
\hline & & Wild type & Mutated & \\
\hline \multirow{2}{*}{ Patients 'age (years) } & Mean (SD) & $54.6(13.0)$ & $57.6(11.7)$ & \multirow{2}{*}{0.63} \\
\hline & Median & 60 & 60 & \\
\hline \multirow{2}{*}{ Patients'sex } & Male & 10 & 11 & \multirow{2}{*}{0.22} \\
\hline & Female & 9 & 4 & \\
\hline \multirow{2}{*}{ Tumor size (cm) } & Mean (SD) & $5.2(2.3)$ & $6.7(2.0)$ & \multirow{2}{*}{0.047} \\
\hline & Median & 5 & 7 & \\
\hline \multirow{2}{*}{ Tumor site (n) } & Central & 14 & 9 & \multirow{2}{*}{0.397} \\
\hline & Peripheral & 5 & 6 & \\
\hline \multirow{2}{*}{ Histological type (n) } & Adenocarcinoma & 16 & 15 & \multirow{2}{*}{0.107} \\
\hline & Squamous cell carcinoma & 3 & 0 & \\
\hline \multirow{2}{*}{ Tumor grade (n) } & Low (grade I and II) & 10 & 8 & \multirow{2}{*}{0.968} \\
\hline & High (grade III and IV) & 9 & 7 & \\
\hline \multirow{3}{*}{ T stage (n) } & $\mathrm{T} 1$ & 4 & 1 & \multirow{3}{*}{0.277} \\
\hline & $\mathrm{T} 3$ & 9 & 11 & \\
\hline & $\mathrm{T} 4$ & 6 & 3 & \\
\hline \multirow{2}{*}{$N$ stage (n) } & $\mathrm{N} 0 / \mathrm{N} 1$ & 11 & 8 & \multirow{2}{*}{0.790} \\
\hline & $\mathrm{N} 2 / \mathrm{N} 3$ & 8 & 7 & \\
\hline \multirow{2}{*}{ M stage (n) } & M0 & 8 & 7 & \multirow{2}{*}{0.790} \\
\hline & M1 & 11 & 8 & \\
\hline
\end{tabular}
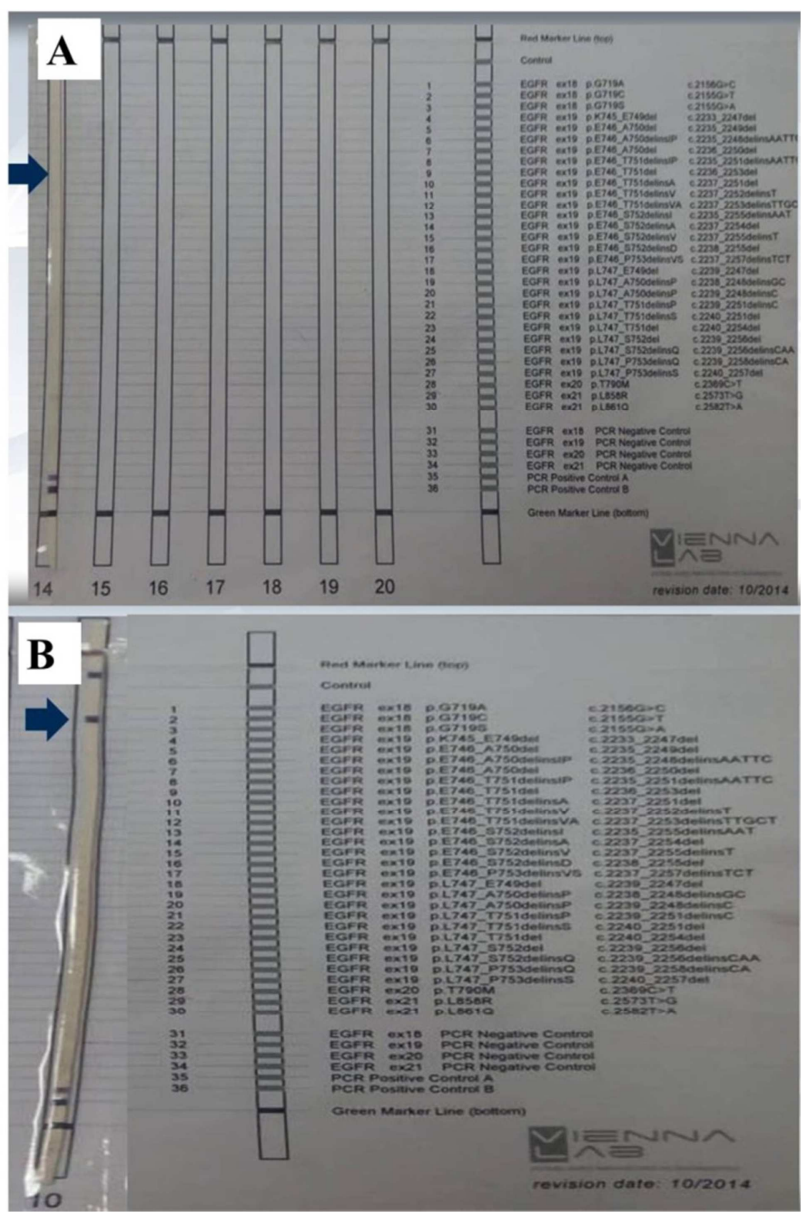

Figure 2. Wide type (A) and mutated Exon 19 (B) of EGFR gene.

\subsection{Treatment and Patients`Survival}

Based on stage of the disease; the investigated patients received either chemotherapy or chemo-irradiation in 19 and 15 cases, respectively. Additional local radiotherapy was directed to sites of metastasis in 8 patients. Regarding chemotherapy regimens; 14 patients were treated with a combination of Vepsede/Carboplatin and 7 patients were treated with a combination of Taxol/Cisplatin. The combinations of Gemcitabine/Carboplatin, Taxol/Carboplatin, Vepsede/Cisplatin were used for 3 patients each and the combinations of Gemcitabine/Cisplatin and Navelbine/Carboplatin and Navelbine/Cisplatin were used for 2, 1 and 1 patients, respectively. The mean $( \pm$ SD) duration of treatment was $13.0( \pm 7.12)$ months and the median duration was 9.0 months. The patients had complete or partial response in 15 patients and stationary or progressive disease in 19 patients. Statistically, mutation of EGFR was associated with stationary or progressive course of lung carcinoma while tumors with wild type EGFR were more frequently responsive to chemotherapy $[\chi 2(1)=3.12, p=0.069$, Figure 3$]$.

The investigated patients were followed up for to 41 months with a mean $( \pm$ SD) and median values of $19.5( \pm 8.6)$ and 20.5 months, respectively. According to life tables, the two-year survival rate for patients with non-small lung cancer of this study was $70.59 \%$ and the overall survival rate was $58.81 \%$ (Figure 4A). Patients that harbored EGFR in their tumors tend to have a relatively worse overall survival rate compared to patients with wild type EGRF (Figure 4B); but the difference did not reach the level of significance (Log-rank; $p=0.136)$. 


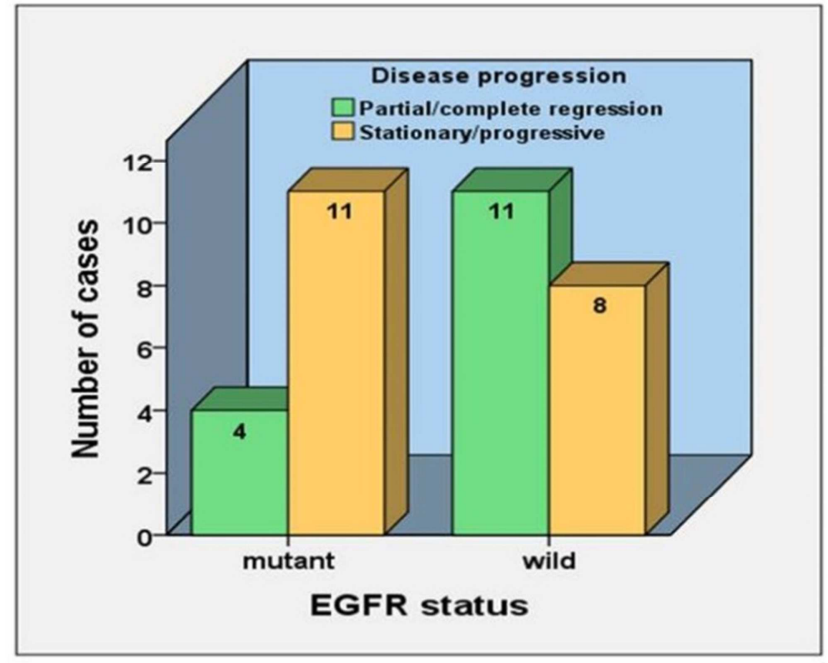

Figure 3. Mutation of EGFR is associated with stationary or progressive course of bronchial carcinoma $(p=0.069)$.

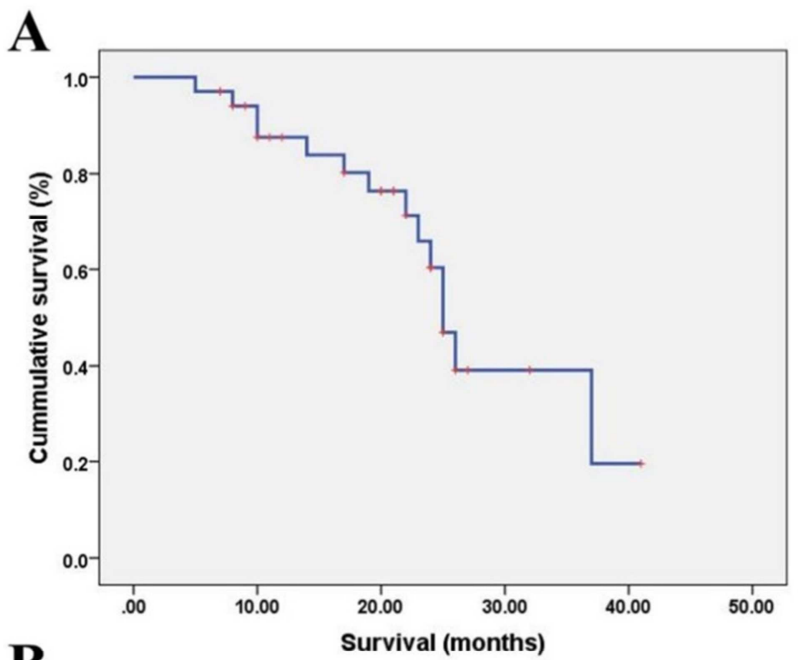

B

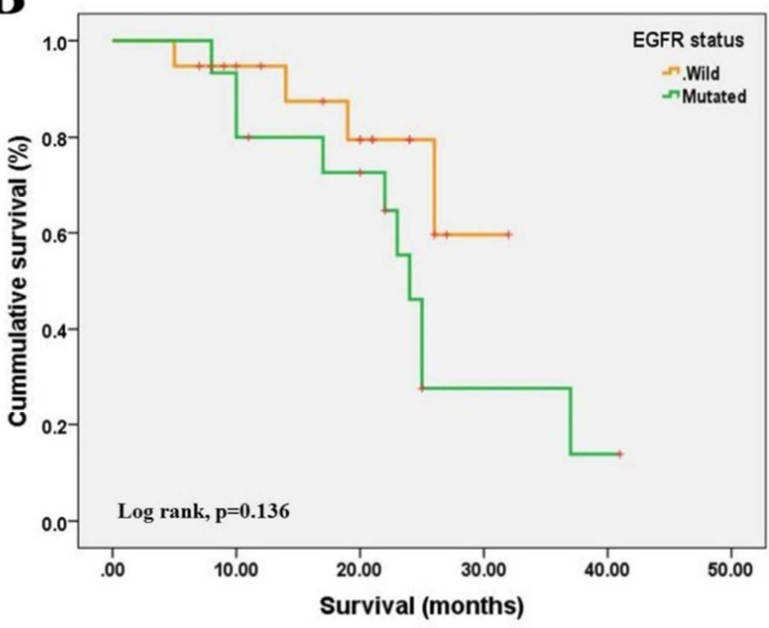

Figure 4. Kaplan-Meier graph for survival of the investigated NSCLC patients (A) and survival in patients with different EGFR status (B).

\section{Discussion}

Association of EGFR mutation with clinic-pathological features and prognosis of NSCLC has been previously reported [5, 6]. In addition, evaluation of EGFR mutation has become the standard care for patients with advanced NSCLC in many countries based on significant improvement of clinical outcome achieved by EGFR inhibitors [11, 12]. It is essential to characterize pattern of NSCLC on molecular basis as lung cancer showed wide genetic heterogeneity among various populations $[13,14]$. This work is the first trial to explore frequency of EGFR mutations in patients with NSCLC in Upper Egypt.

EGFR mutation was recorded in $44.1 \%$ of the tested tumors which is comparable to reports of other nations. Previous reports demonstrated that mutations of EGFR in lung cancer had a wide variation among different populations. Vietnam and Taiwan had the highest rates of EGFR mutations representing $64 \%$ and $62.1 \%$ of the investigated NSCLC; respectively; followed by China (37\%) and Japan (29\%) while USA and Australia recorded the least rates of mutations; $14 \%$ and $7 \%$, respectively. In India, the rate of EGFR mutation ranged between $23.2 \%$ and $51.8 \%$ [13-16]. In general, the rate of EGFR mutation is higher in Asian population (47\%) compared to Caucasian (13\%) [17]. The authors have attributed these different rates to race and ethnic factors. In the current study; mutations of EGFR were detected mainly in chromosome 18 (4 tumors), chromosome 20 (7 tumors) and chromosome 21 (7 tumors); which is compatible to previous findings. Exon sequencing of genomic DNA in lung cancer revealed missense and deletion of EGFR in a total of 16 tumors, all within exons 18 through 21 of kinase domain [6]. In another study, mutations of EGFR involved predominantly chromosome 18 and 21 in NSCLC [18].

In this cohort, the patients`age ranged between 30 to 77 years with a mean age of 55.9 years and $61.0 \%$ of the patients were males; findings that are compatible with previous data $[19,20]$. In contrast to several studies that had documented higher frequency of EGFR mutations in female compared to males and higher rates in older patients [18-22]; there was no significant difference of EGFR mutations among both sexes or association of EGFR mutation with patients' age in this study. Ethnic differences and small number of female patients are probable explanations for these findings. Although EGFR mutation is higher in Indian females compared to males; both sexes have the same rates in Caucasians which ensures the relationship of ethnity with mutations of EGFR gene [16].

The three investigated squamous cell carcinomas in this study had wild type EGFR gene while nearly half of the investigated adenocarcinomas harbored EGFR mutations. Several studies documented the high frequency of EGFR mutations in pulmonary adenocarcinoma [6, 7, 12, 23, 24]. Perez-Moreno P et al [25] and Chougule A et al [16] reported mutations of EGFR gene in $5.8 \%$ and $3.8 \%$ of the investigated squamous cell variant of NSCLC; respectively. They ensured that some of these tumors are adeno-squamous rather than pure squamous cell carcinomas.

Mutation of EGFR is significantly more frequent in large sized tumors, but it was not correlated with tumor site, grade, T stage, nodal stage or status of distant metastasis (Table 2). It has been reported that EGFR mutations are significantly 
associated with moderately differentiated pulmonary adenocarcinomas [26] and with visceral pleural invasion in NSCLC [27]. There was no reported correlation of EGFR mutations with tumor stage, lymph node metastasis or distant metastasis $[21,22,26]$.

The two-year survival rate of the investigated patients was $70.59 \%$ and the overall survival rate was $58.8 \%$ (Figure 4A). Patients who harbored EGFR mutations in their tumors tend to have a relatively worse overall survival compared to wild type EGRF patients (Figure 4B). In contrast; several published studies over the last decades ensured that patients with EGFR mutations are candidate for gefitinib; an EGFR inhibitor with significant improvement of progression-free survival [7, 8, 10 $12,28]$. Patients in this study were treated with conventional chemo- or chemo-radiotherapy as target therapy (EGFR inhibitors and tyrosine kinase inhibitors) are not available in Upper Egypt for economic reasons. Furthermore; $60 \%$ of the investigated patients had local T3 stage, $42 \%$ has N2 stage and $56 \%$ had distant metastasis or metastasis to contralateral lung; implying advanced disease stage.

\section{Limitations}

Finally, are some limitations First, this is a retrospective study with limited tissue obtained from the patients. Evaluation of EGFR mutation was not performed at time of initial diagnosis of NSCLC; which is a critical parameter to select first-line treatment. Second, the study was performed in patients with different clinical settings and different stages of distant metastasis at time of presentation. The small sample size is a third limiting factor that required careful interpretation of obtained findings.

\section{Conclusions}

The rate of EGFR mutation in NSCLC in Upper Egypt is relatively high and it is mainly located on chromosomes 18 and 21. These mutations are more frequent in large sized tumors and in adenocarcinoma variants of the tumors. There is no association of EGFR mutation with patients` age or gender, with tumor grade, lymph node stage or distant metastasis. In absence of target therapy, RGFR mutation is correlated with stationary/progressive course and poor survival of NSCLC.

\section{Conflict of Interest}

All the authors do not have any possible conflicts of interest.

\section{References}

[1] Lin L, Bivona TG. Mechanisms of Resistance to Epidermal Growth Factor Receptor Inhibitors and Novel Therapeutic Strategies to Overcome Resistance in NSCLC Patients. Chemother Res Pract. 2012; 2012: 817297. doi: 10.1155/2012/817297.

[2] Ibrahim AS, Khaled HM, Mikhail NN, Baraka H, Kamel H.
Cancer incidence in egypt: results of the national population-based cancer registry program. J Cancer Epidemiol. 2014; 2014: 437971. doi: 10.1155/2014/437971.

[3] Mendelsohn J, Baselga J. Epidermal growth factor receptor targeting in cancer. Semin Oncol. 2006; 33 (4): 369-85.

[4] Scagliotti GV, Selvaggi G, Novello S, Hirsch FR. The biology of epidermal growth factor receptor in lung cancer. Clin Cancer Res 2004; 10 (12Pt2): 4227s-32s.

[5] Lynch TJ, Bell DW, Sordella R, Gurubhagavatula S, Okimoto $\mathrm{RA}$, Brannigan BW, et al. Activating mutations in the epidermal growth factor receptor underlying responsiveness of non-small-cell lung cancer to gefitinib. N Engl J Med. 2004; 350 (21): 2129-39.

[6] Paez JG, Janne PA, Lee JC, Tracy S, Greulich H, Gabriel S, et al. EGFR mutations in lung cancer: correlation with clinical response to gefitinib therapy. Science. 2004; 304 (5676): 1497-500.

[7] Mok TS, Wu YL, Thongprasert S, Yang CH, Chu DT, Saijo N, et al. Gefitinib or carboplatin-paclitaxel in pulmonary adenocarcinoma. N Engl J Med. 2009; 361 (10): 947-57.

[8] Rosell R, Carcereny E, Gervais R, Vergnenegre A, Massuti B, Felip E, et al. Erlotinib versus standard chemotherapy as first-line treatment for European patients with advanced EGFR mutation-positive non-small-cell lung cancer (EURTAC): a multicentre, open-label, randomised phase 3 trial. Lancet Oncol. 2012; 13 (3): 239-46.

[9] Inoue A, Kobayashi K, Usui K, Maemondo M, Okinaga S, Mikami I, et al. First-line gefitinib for patients with advanced non-small-cell lung cancer harboring epidermal growth factor receptor mutations without indication for chemotherapy. J Clin Oncol. 2009; 27 (9): 1394-400.

[10] Maemondo M, Inoue A, Kobayashi K, Sugawara S, Oizumi S, Isobe $\mathrm{H}$, et al. Gefitinib or chemotherapy for non-small-cell lung cancer with mutated EGFR. N Engl J Med. 2010; 362 (25): 2380-88.

[11] Lindeman NI, Cagle PT, Beasley MB, Chitale DA, Dacic S, Giaccone $\mathrm{G}$, et al. Molecular testing guideline for selection of lung cancer patients for EGFR and ALK tyrosine kinase inhibitors: guideline from the College of American Pathologists, International Association for the Study of Lung Cancer, and Association for Molecular Pathology. Arch Pathol Lab Med. 2013; 137 (6): 828-60.

[12] Haaland B, Tan PS, de Castro G, Jr., Lopes G. Meta-analysis of first-line therapies in advanced non-small-cell lung cancer harboring EGFR-activating mutations. J Thorac Oncol. 2014; 9 (6): 805-11.

[13] Graham RP, Treece AL, Lindeman NI, Vasalos P, Shan M, Jennings LJ, et al. Worldwide Frequency of Commonly Detected EGFR Mutations. Arch Pathol Lab Med. 2018; 142 (2): 163-167.

[14] Shi Y, Au JS, Thongprasert S, Srinivasan S, Tsai CM, Khoa MT, et al. A prospective, molecular epidemiology study of EGFR mutations in Asian patients with advanced non-small-cell lung cancer of adenocarcinoma histology (PIONEER). J Thorac Oncol. 2014; 9 (2): 154-62.

[15] Sahoo R, Harini VV, Babu VC, Patil Okaly GV, Rao S, Nargund A, et al. Screening for EGFR mutations in lung cancer, a report from India. Lung Cancer. 2011; 73 (3): 316-19. 
[16] Chougule A, Prabhash K, Noronha V, Joshi A, Thavamani A, Chandrani P, et al. Frequency of EGFR mutations in 907 lung adenocarcioma patients of Indian ethnicity. PLoS One. 2013, 8 (10): e76164.

[17] Sekine I, Yamamoto N, Nishio K, Saijo N. Emerging ethnic differences in lung cancer therapy. Br J Cancer. 2008; 99 (11): 1757-62.

[18] Veldore VH, Rao RM, Kakara S, Pattanayak S, Tejaswi R, Sahoo R, et al. Epidermal growth factor receptor mutation in non-small-cell lung carcinomas: a retrospective analysis of 1036 lung cancer specimens from a network of tertiary cancer care centers in India. Indian J Cancer. 2013; 50 (2): 87-93.

[19] Enewold L, Thomas A. Real-World Patterns of EGFR Testing and Treatment with Erlotinib for Non-Small Cell Lung Cancer in the United States. PLoS One. 2016; 11 (6): e0156728.

[20] Tomizawa Y, Iijima H, Sunaga N, Sato K, Takise A, Otani Y, et al. Clinicopathologic significance of the mutations of the epidermal growth factor receptor gene in patients with non-small cell lung cancer. Clin Cancer Res. 2005; 11 (19Pt1): 6816-22.

[21] Wang H, Zhang W, Wang K, Li X. Correlation between EML4-ALK, EGFR and clinicopathological features based on IASLC/ATS/ERS classification of lung adenocarcinoma. Medicine (Baltimore). 2018; 97 (26): e11116.

[22] Choi YH, Lee JK, Kang HJ, Lee TS, Kim HR, Kim CH, et al. Association between age at diagnosis and the presence of EGFR mutations in female patients with resected non-small cell lung cancer. J Thorac Oncol. 2010, 5 (12): 1949-52.
[23] Chapman AM, Sun KY, Ruestow P, Cowan DM, Madl AK. Lung cancer mutation profile of EGFR, ALK, and KRAS: Meta-analysis and comparison of never and ever smokers. Lung Cancer. 2016; 102: 122-34.

[24] Miyamae Y, Shimizu K, Hirato J, Araki T, Tanaka K, Ogawa H, et al. Significance of epidermal growth factor receptor gene mutations in squamous cell lung carcinoma. Oncol Rep. 2011, 25 (4): 921-28.

[25] Perez-Moreno P, Brambilla E, Thomas R, Soria JC. Squamous cell carcinoma of the lung: molecular subtypes and therapeutic opportunities. Clin Cancer Res. 2012; 18 (9): 2443-51.

[26] Wang K, Gong H, Li X, Yang Z, Cao P, Wand C, et al. Relationship between histopathologic characteristics and epidermal growth factor receptor mutation in lung adenocarcinoma. Zhonghua Bing Li Xue Za Zhi. 2015; 44 (3): $170-4$.

[27] Shi J, Yang Y, Zhao Y, Zhu J, Song X, Jiang G. EGFR mutations are significantly associated with visceral pleural invasion development in non-small-cell lung cancer patients. Cancer Manag Res. 2019; 11: 1945-57.

[28] Mitsudomi T, Morita S, Yatabe Y, Negoro S, Okamoto I, Tsurutani J, et al. Gefitinib versus cisplatin plus docetaxel in patients with non-small-cell lung cancer harbouring mutations of the epidermal growth factor receptor (WJTOG3405): an open label, randomised phase 3 trial. Lancet Oncol. 2010; 11 (2): 121-8. 